\section{BMJ Open} Ophthalmology

\section{Physical inactivity in relation to self- rated eyesight: cross-sectional analysis from the English Longitudinal Study of Ageing}

To cite: Smith L, Timmis MA, Pardhan S, et al. Physical inactivity in relation to self-rated eyesight: crosssectional analysis from the English Longitudinal Study of Ageing. BMJ Open Ophth 2016;1:e000046. doi:10.1136/bmjophth-2016000046

- Prepublication history and additional material is available. To view please visit the journal (http://dx.doi.org/ 10.1136/bmjophth-2016000046)

Received 27 September 2016

Revised 09 November 2016 Accepted 01 December 2016

\section{(a) CrossMark}

${ }^{1}$ The Cambridge Centre for Sport and Exercise Sciences, Department of Life Sciences, Anglia Ruskin University, Cambridge, UK

${ }^{2}$ Vision and Eye Research Unit, Anglia Ruskin University, Cambridge, UK ${ }^{3}$ Visual Function and Physiology Research Group, Anglia Ruskin University, Cambridge, UK

${ }^{4}$ School Sport, Exercise \& Health Sciences, Loughborough University, Loughborough, UK

Correspondence to Dr Lee Smith; lee.smith@ anglia.ac.uk

\section{ABSTRACT}

Background: To assess the cross-sectional association between self-rated eyesight and physical activity behaviour in a large general population sample of older English adults.

Methods: Analyses of data from the English Longitudinal Study of Ageing. Participants provided information on self-rated eyesight (categorised as: excellent/very good/good/fair-poor) and their own physical activity levels (categorised as: inactive/ moderate only at least $1 /$ week, vigorous at least $1 /$ week). Associations between self-rated eyesight and physical activity levels were examined using logistic regression.

Results: A total of 6634 participants (mean age $65.0 \pm 9.2$ years) were included in the analyses. In adjusted logistic regression models, those with fairpoor and good eyesight were significantly more likely to be inactive than those who reported excellent eyesight (OR 2.07, $95 \% \mathrm{Cl} 1.58$ to 2.72; OR 1.59, 1.27 to 1.99 , respectively).

Conclusion: In this sample of older English adults, those with self-rated fair-poor vision were over twice as likely to be physically inactive than those who reported having excellent vision. When consistent data have emerged, interventions to increase physical activity in those who have poor eyesight are needed.

\section{INTRODUCTION}

Physical activity may be defined as any bodily movement caused by contraction of skeletal muscle that requires energy expenditure. ${ }^{1}$ In older adults ( $\geq 50$ years), regular participation in physical activity has been found to be associated with reduced risk of cardiovascular disease (CVD), diabetes and certain cancers, as well as prevention of falls and greater independence. ${ }^{2-7}$ In light of this knowledge, physical activity guidelines for older adults have been developed. It is recommended that older adults achieve at least $150 \mathrm{~min}$ of moderate-intensity activity over a week. ${ }^{8}$ Despite this, levels of physical activity in older adults are generally lower

\section{Key messages}

What is already known about this subject?

In the visually impaired, there is a lack of access to recreational and athletic programmes, and help or encouragement in developing suitable and safe physical recreation skills and habits.

- This population experiences activity limitations in walking, and environmental barriers such as transport and lack of accessible exercise equipment.

- No research has been carried out on the association between eyesight and physical activity levels in large general population cohorts.

What are the new findings?

- In a population-based sample of older English adults, those with self-rated fair-poor vision were over twice as likely to be physically inactive than those who reported having excellent vision.

How might these results change the focus of research or clinical practice?

When consistent data have emerged, interventions to increase physical activity in those who have poor eyesight are needed.

than the guidelines and there is an overall trend for decreasing levels of vigorous physical activity as adults age. ${ }^{9}{ }^{10}$ Indeed, Smith et $a l^{9}{ }^{9}$ in a sample of 5022 older English adults (mean age 61 years), found that over a 10-year period there was an overall trend for increasing levels of inactivity and a reduction in vigorous activity.

Correlates of physical activity behaviour in adults and older adults are well documented and low levels of physical activity is known to be a greater problem among persons with disabilities. ${ }^{11}$ One particular disability that may present itself as a key barrier to physical activity in older adults is reduced 
eyesight. ${ }^{12}$ This is of particular concern as approximately two million people in the UK are living with sight loss (defined here as partial sight or blindness in the better seeing eye) and the prevalence of sight loss is on the rise, ${ }^{13}$ likely owing to the ageing UK population. ${ }^{14}$ Those who are visually impaired have a high risk of CVD mortality ${ }^{15}$ and a key determinant of CVD is low levels of physical activity. A large body of literature exists on the association between visual impairment and physical activity levels, but only in children and adolescents. ${ }^{12}$ In a sample of 987 Canadian youth, the percentage of children and adolescents with chronic medical conditions and hearing impairments who were active ( $47 \%$ and $53 \%$, respectively) were significantly higher than children and adolescents with physical disorders (26\%) or visual impairment $(27 \%) .{ }^{16}$ This is of concern as research suggests that childhood activity behaviours track into adulthood. ${ }^{17-}$

${ }^{19}$ For example, in a study of 6458 children, those who participated in sports at 10 years of age were significantly more likely to participate in physical activity at age 42 (RR 1.10; 95\% CI 1.01 to 1.19 ). ${ }^{18}$ Children and adolescents with physical disorder and visual impairment may be an at-risk group of lifelong physical inactivity.

It has been suggested that in the visually impaired there is a lack of access to recreational and athletic programmes, and help or encouragement in developing suitable and safe physical recreation skills and habits. ${ }^{12}$ Moreover, this population experiences activity limitations in walking, and environmental barriers such as transport and lack of accessible exercise equipment can hamper a person's ability to be physically active. ${ }^{12}$ ${ }^{20}$ Other research in this population has focused on sociodemographic correlates of physical activity. ${ }^{21}$ To the best of our knowledge, no research has been carried out on the association between eyesight and physical activity levels in a large-scale general population-based cohort study of older English adults. Visual impairment is more common in older age groups, ${ }^{13}$ such that older adults may have experienced normal eyesight throughout their adulthood but suffer deterioration with age, possibly resulting in less confidence and/or ability to undertake certain activities. We hypothesise that poor self-perceived eyesight will be associated with low levels of physical activity.

\section{METHODS}

The English Longitudinal Study of Ageing (ELSA) is an on-going cohort study containing a nationally representative sample of the English population living in households. The cohort consists of men and women born on or before 29 February $1952 .{ }^{22}$ For the purpose of the present analysis, data collected during wave 4 (2008-2010) were used. Participants gave full written informed consent to participate in the study and ethical approval was obtained from the London Multicentre Research Ethics Committee.

\section{Exposure: self-rated eyesight}

To assess self-rated eyesight, participants were asked 'Is your eyesight (using glasses or corrective lenses; if you use them) excellent/very good/good/fair/ or poor'. Based on response options, the participants were then categorised into one of four groups (excellent/very good/good/fair-poor). Participants were also asked 'How good is your eyesight for seeing things at a distance, like recognising a friend across the street' and 'How good is your eyesight for seeing things up close, like reading ordinary newspaper print'. Response options (excellent/very good/good/fair-poor) were categorised as above.

\section{Outcome: physical activity}

Participants were asked how often they took part in vigorous, moderate and low-intensity physical activity, as previously described. ${ }^{9}$ Response options were: more than once a week, once a week, one to three times a month, hardly ever/never. Based on response options, the participants were then categorised into one of three groups (inactive, moderate only at least 1/ week, vigorous at least 1 /week). This measure has been shown to have convergent validity in grading a plethora of psychosocial, physical and biochemical risk factors. ${ }^{23-25}$

\section{Covariates}

Age and sex were self-reported. Trained interviewers asked questions on smoking (current, previous or non-smoker), alcohol intake (daily, at least once a week, monthly, rarely, never), depressive symptoms (using the eight-item Centre for Epidemiological Studies Depression Scale), history of CVD (angina, heart attack, stroke, hypertension), history of diabetes and history of eye disease (glaucoma, diabetic eye disease, macular degeneration, cataract). Disabilities were assessed based on participant's responses to interviewers' questions on perceived difficulties in six basic activities of daily living (ADLs), such as difficulty dressing, and seven instrumental ADLs (IADLs), such as preparing a hot meal. Participants with difficulties in one or more activities were considered to have some degree of disability. Research nurses measured participants' body weight using Tanita electronic scales, participants were measured without shoes and in light clothing. Height was measured using a stadiometer with the Frankfort plane in the horizontal position. Body mass index (BMI) was calculated using the standard formula (weight $(\mathrm{kg}) /$ height $^{2}(\mathrm{~m})$ ).

\section{Analysis}

Characteristics of the study population were summarised using descriptive statistics. Associations between self-rated eyesight and self-reported physical activity were examined using logistic regression models, 
adjusted for prespecified covariates based on existing literature. Statistical significance was set at $p=0.05$. All analyses were conducted in SPSS V.21.

\section{RESULTS}

The initial sample comprised 10603 participants, although exclusion of participants with missing data resulted in a final analytical sample of 6634 participants (mean age 65.0 \pm 9.2 years). Participants excluded were slightly older (65.4 vs 65.0 years, $\mathrm{p}=0.04$ ) and more likely to report poor-fair eyesight $(15.2 \%$ vs $10.6 \%, \mathrm{p}=0.001)$ and limitations in ADLs/ IADLs $(33.1 \%$ vs $22.8 \%, \mathrm{p}=0.001)$ than the analytical sample.

A total of $16.2 \%, 35.6 \%, 37.7 \%$ and $10.6 \%$ of the sample rated their eyesight as excellent, very good, good and fair-poor, respectively. Those with fair-poor self-rated eyesight were older (mean 67.8 years) and more likely to be female $(59.6 \%)$ with the highest prevalence of obesity (BMI $\left.\geq 30 \mathrm{~kg} / \mathrm{m}^{2} ; 35.3 \%\right)$, smoking (18.3\%), depressive symptoms (Centre for Epidemiological Studies Depression Scale score $\geq 4$, $23.6 \%$ ), disabilities (46.3\%), history of CVD (44.7\%), history of diabetes $(11.1 \%)$ and history of eye disease, but reported less frequent alcohol intake (table 1).

Compared with other self-rated eyesight groups, the group 'excellent eyesight' contained the greatest proportion of participants reporting participation in moderate and vigorous activities and the smallest proportion of inactive participants. The group 'fairpoor eyesight' contained the greatest proportion of inactive participants and the smallest proportion of those vigorously active (table 1 ).

In adjusted logistic regression models, those with fairpoor and good eyesight were significantly more likely to be inactive than those who reported excellent eyesight (OR 2.07, 95\% CI 1.58 to 2.72; OR 1.59, 1.27 to 1.99 , respectively; table 2). Similar findings were found for

Table 1 Descriptive characteristics of the sample $(n=6634)$

\begin{tabular}{|c|c|c|c|c|}
\hline \multirow[t]{2}{*}{ Variable } & \multicolumn{4}{|c|}{ Self-rated eyesight } \\
\hline & $\begin{array}{l}\text { Excellent } \\
(n=1078)\end{array}$ & Very good $(n=2359)$ & Good $(n=2497)$ & Fair-poor $(n=700)$ \\
\hline Age, mean \pm SD & $63.2 \pm 8.5$ & $64.4 \pm 8.7$ & $65.5 \pm 9.2$ & $67.8 \pm 10.5$ \\
\hline Sex, $n$ (\% men) & $534(49.5)$ & $1075(45.6)$ & $1091(43.7)$ & $283(40.4)$ \\
\hline \multicolumn{5}{|l|}{ Physical activity, n (\%) } \\
\hline Inactive & $120(11.1)$ & $343(14.5)$ & $531(21.3)$ & $243(34.7)$ \\
\hline Moderate at least $1 /$ week & $547(50.7)$ & 1138 (48.2) & $1233(49.4)$ & $340(48.6)$ \\
\hline Vigorous at least $1 /$ week & $411(38.1)$ & $878(37.2)$ & $733(29.4)$ & $117(16.7)$ \\
\hline Obesity (BMI $\geq 30$ kg/m²), n (\%) & $313(29.0)$ & $718(30.4)$ & $790(31.6)$ & $247(35.3)$ \\
\hline Current smoker, n (\%) & $115(10.7)$ & $250(10.6)$ & $342(13.7)$ & $128(18.3)$ \\
\hline \multicolumn{5}{|l|}{ Alcohol intake, n (\%) } \\
\hline$\geq 5$ times a week & $297(27.6)$ & $549(23.3)$ & $576(23.1)$ & 118 (16.9) \\
\hline 1-4 times a week & 452 (41.9) & 989 (41.9) & 947 (37.9) & 247 (35.3) \\
\hline Monthly & $177(16.4)$ & $461(19.5)$ & $471(18.9)$ & 135 (19.3) \\
\hline Rarely/never & $152(14.1)$ & $360(15.3)$ & $503(20.1)$ & $200(28.6)$ \\
\hline $\begin{array}{l}\text { Depressive symptoms (CES-D score } \geq 4 \text { ), } \\
n(\%)\end{array}$ & $82(7.6)$ & $193(8.2)$ & $374(15.0)$ & 165 (23.6) \\
\hline Disabilities* (at least one), n (\%) & $160(14.8)$ & $410(17.4)$ & $622(24.9)$ & $324(46.3)$ \\
\hline History of CVD†, n (\%) & $282(26.2)$ & $670(28.4)$ & $814(32.6)$ & $313(44.7)$ \\
\hline History of diabetes, n (\%) & $70(6.5)$ & $112(4.7)$ & $145(5.8)$ & $78(11.1)$ \\
\hline \multicolumn{5}{|l|}{ History of eye diseases, n (\%) } \\
\hline Glaucoma & $15(1.4)$ & $31(1.3)$ & $53(2.1)$ & $18(2.6)$ \\
\hline Diabetic eye disease & $1(0.1)$ & $9(0.4)$ & $17(0.7)$ & $7(1.0)$ \\
\hline Macular degeneration & $3(0.3)$ & $20(0.8)$ & $22(0.9)$ & $30(4.3)$ \\
\hline Cataract & $60(5.6)$ & $158(6.7)$ & $267(10.7)$ & $157(22.4)$ \\
\hline
\end{tabular}

${ }^{*}$ Recorded from ADLs/IADLs.

†Includes angina, coronary heart disease, stroke and hypertension.

ADLs, activities of daily living; BMI, body mass index; CES-D, Centre for Epidemiological Studies Depression Scale; CVD, cardiovascular disease; IADLs, instrumental ADLs;. 
Table 2 Logistic regression analyses to examine the association between self-rated eyesight and physical inactivity $(n=6634)$

\begin{tabular}{llll}
\hline Self-rating & $\begin{array}{l}\text { Self-rated eyesight } \\
\text { OR }\end{array}$ & $\begin{array}{l}\text { Recognition of friends across street } \\
\text { OR }^{\star}(\mathbf{9 5} \% \mathbf{C l})\end{array}$ & $\begin{array}{l}\text { Reading ordinary newspaper } \\
\text { OR }^{\star}(\mathbf{9 5 \%} \text { Cl) }\end{array}$ \\
\hline Excellent & $1.0($ Ref $)$ & 1.0 & 1.0 \\
Very good & $1.22(0.96$ to 1.54$)$ & $1.08(0.90$ to 1.30$)$ & $1.04(0.85$ to 1.27$)$ \\
Good & $1.59(1.27$ to 1.99$)$ & $1.28(1.06$ to 1.55$)$ & $1.31(1.06$ to 1.59$)$ \\
Fair-poor & $2.07(1.58$ to 2.72$)$ & $1.89(1.44$ to 2.49$)$ & $1.51(1.17$ to 1.97$)$
\end{tabular}

*Adjusted for age, sex, smoking, alcohol, depressive symptoms, ADLs/IADLs, body mass index, CVD, diabetes, eye diseases. ADLs, activities of daily living; IADLs, instrumental ADLs; CVD, cardiovascular disease.

the variable 'recognition of friends across street' and 'reading ordinary newspaper' (table 2). There was no association between self-reported eye diseases and physical inactivity $(\mathrm{OR}=0.88,95 \%$ CI 0.72 to 1.07 , $\mathrm{p}=0.18)$.

\section{DISCUSSION}

This is the first study to investigate the association between self-rated vision and physical activity behaviour in a large general population sample of older adults in the UK. Participants who reported having fair-poor eyesight were more than twice as likely to be inactive than those reporting excellent eyesight. Many of the covariates used in this analysis (ie, depressive symptoms, ADLs/IADLs, BMI, CVD, diabetes) have been related to physical activity in our previously published work from ELSA. ${ }^{23} 2627$ Thus, the present findings are remarkable in that associations with eyesight remained robust after accounting for these important confounders. These findings support previous work in children and adolescents and recent data from Sport England that states that only 9.8\% of visually impaired people are active once a week compared with approximately $30 \%$ of those who are not visually impaired.

Low levels of physical activity in those reporting fairpoor eyesight is of concern as this population may be at an increased risk of non-communicable disease, such as higher risk of cancer ${ }^{28}$ and also have associated risk factors such as higher smoking rates, ${ }^{29}$ independent of physical activity. Moreover, those who are visually impaired often report having a low quality of life. For example, one study concluded that visual impairment has a substantial impact on the quality of life compared with other chronic conditions, reducing quality of life more than diabetes, coronary syndrome and hearing impairments. Higher levels of physical activity are consistently associated with higher quality of life. ${ }^{30}$

Data from the present study must be interpreted with caution. Measures of eyesight and physical activity were self-reported and thus may have introduced bias. For example, participants may have reported higher levels of physical activity and better vision than would be measured objectively owing to fear of being judged, however such bias is likely to be systematic. The present analysis is cross-sectional in design, therefore the causal direction of the observed association cannot be inferred. It is unclear whether poor eyesight causes physical inactivity or if physical inactivity causes poor eyesight. It is likely to be a combination of both. Participants with poor eyesight may lack confidence to undertake physical activity particularly of a vigorous nature and that they may feel unsafe/insecure during this activity. On the other hand, lack of physical activity may increase the likelihood of other metabolic diseases such as diabetes which in turn may lead to severe sightthreatening issues if not controlled adequately. Regular exercise has been shown to increase antioxidant enzyme activity and increase resistance to oxidative stress which is thought to be one of the key components in the pathogenesis of age-related macular degeneration. ${ }^{31}{ }^{32}$ Studies have also shown a protective association between physical activity and age-related macular degeneration but others have not. ${ }^{33-35}$ Animal studies provide some evidence to support that physical activity may aid in the reduction of eye disease. One study using mice demonstrated that aerobic exercise is neuroprotective for retinal degeneration. ${ }^{36}$ Prospective and controlled intervention studies investigating the relationship between self-rated eyesight and physical activity, in human samples, are now needed.

In the present study, self-reported eye disease per se was not associated with self-reported physical activity but self-rated eyesight was. The reason for this difference is yet to be explored. We encourage future research in this area. Participants excluded from our analysis contained a higher proportion reporting poorer eyesight and limitations in ADLs/IADLs, thus our results may be conservative. We cannot discount residual confounding that may have explained the associations between eyesight and physical activity although we attempted to control for a wide range of clinical and behavioural covariates. 


\section{CONCLUSIONS}

In this sample of older English adults those with selfrated fair-poor vision were over twice as likely to be physically inactive than those who reported having excellent vision. When consistent data has emerged, interventions to increase physical activity in those who have poor eyesight are needed. Interventions may wish to overcome common exercise barriers in this population such as transport and lack of accessible exercise equipment.

Contributors LS designed the study, conceived the idea and wrote the paper. MAT, SP, KL and JJ designed the study, conceived the idea and approved the final version of the manuscript. MH designed the study, conceived the idea, analysed the data and approved the final version of the manuscript.

Competing interests None declared.

Provenance and peer review Not commissioned; externally peer reviewed.

Open Access This is an Open Access article distributed in accordance with the Creative Commons Attribution Non Commercial (CC BY-NC 4.0) license, which permits others to distribute, remix, adapt, build upon this work noncommercially, and license their derivative works on different terms, provided the original work is properly cited and the use is non-commercial. See: http:// creativecommons.org/licenses/by-nc/4.0/

\section{REFERENCES}

1. Caspersen CJ, Powell KE, Christenson GM. Physical activity, exercise, physical fitness: definitions and distractions for healthrelated research. Public Health Rep 1985;100:126-31.

2. Mazzeo RS, Cavanagh P, Evans WJ, et al. Exercise and physical activity for older adults. Med Sci Sports Exerc 1998;30:992-1008.

3. Pullen C, Walker SN, Fiandt K. Determinants of health-promoting lifestyle behaviors in rural older women. Fam Community Health 2001;24:49-72.

4. Keysor JJ. Does late-life physical activity or exercise prevent or minimize disablement? A critical review of the scientific evidence. Am J Prev Med 2003;25:129-36.

5. Stein CJ, Colditz GA. Modifiable risk factors for cancer. Br J Cancer 2004;90:299-303.

6. DiPietro L. The epidemiology of physical activity and physical function in older people. Med Sci Sports Exerc 1996;28:596-600.

7. Buchner DM, Wagner EH. Preventing frail health. Clin Geriatr Med 1992;8:1-17.

8. Department of Health. Physical activity guidelines for older adults UK. 2011

9. Smith L, Gardner B, Fisher A, et al. Patterns and correlates of physical activity behaviour over 10 years in older adults: prospective analyses from the English Longitudinal Study of Ageing. BMJ Open 2015;5:e007423.

10. Public Health England. Everybody active every day. An evidence based approach to physical activity. UK: Public Health England, 2014

11. U.S. Department of Health and Human Services. Physical activity and health: A report of the Surgeon General, Executive Summary. Washington, DC: U.S. Department of Health and Human Services, 1996.

12. Capella-McDonnall $M$. The need for health promotion for adults who are visually impaired. J Vis Impair Blind 2007;101:133-45.

13. Access Economics. Future sight loss UK: The economic impact of partial sight and blindness in the UK adult population. UK: RNIB, 2009.
14. Owen CG, Jarrar Z, Wormald R, et al. The estimated prevalence and incidence of late stage age related macular degeneration in the UK. Br J Ophthalmol 2012;96:752-6.

15. Rajala U, Pajunpaa $\mathrm{H}$, Koskela $\mathrm{P}$, et al. High cardiovascular disease mortality in subjects with visual impairment caused by diabetic retinopathy. Diabetes Care 2000;23:957-61.

16. Longmuir $\mathrm{PE}, \mathrm{Bar}-\mathrm{Or} \mathrm{O}$. Factors influencing the physical activity levels of youths with sensory disabilities. Adapt Phys Activ $Q$ 2000;17:40-53.

17. Smith L, Gardner B, Hamer M. Childhood correlates of adult TV viewing time: a 32-year follow-up of the 1970 British Cohort Study. $J$ Epidemiol Community Health 2015;69:309-13.

18. Smith L, Gardner B, Aggio D, et al. Association between participation in outdoor play and sport at 10 years old with physical activity in adulthood. Prev Med 2015;74:31-5.

19. Smith L, Fisher A, Hamer M. Prospective association between objective measures of childhood motor coordination and sedentary behaviour in adolescence and adulthood. Int J Behav Nutr Phys Act 2015b;12:75

20. Phoenix C, Griffin M, Smith B. Physical activity among older people with sight loss: a qualitative research study to inform policy and practice. Public Health 2015;129:124-30.

21. HaegeleJ, Zhu X, Lee J, et al. Physical activity for adults with visual impairments: impact of sociodemographic factors. Eur J Adapt Phys Activity 2016:9:3-14.

22. Steptoe A, Breeze E, Banks J, et al. Cohort profile: the English longitudinal study of ageing. Int $\mathrm{J}$ Epidemiol 2013;42:1640-8.

23. Hamer M, Lavoie KL, Bacon SL. Taking up physical activity in later life and healthy ageing: the English longitudinal study of ageing. $\mathrm{Br} \mathrm{J}$ Sports Med 2014;48:239-43.

24. Hamer M, Stamatakis E. Screen-based sedentary behavior, physical activity, and muscle strength in the English longitudinal study of ageing. PLOS One 2013;8:e66222.

25. Hamer M, Stamatakis E. Prospective study of sedentary behavior, risk of depression, and cognitive impairment. Med Sci Sports Exerc 2014;46:718-23.

26. Demakakos $\mathrm{P}$, Hamer M, Stamatakis E, et al. Low-intensity physical activity is associated with reduced risk of incident type 2 diabetes in older adults: evidence from the English Longitudinal Study of Ageing. Diabetologia 2010;53:1877-85.

27. Hamer M, de Oliveira C, Demakakos P. Non-exercise physical activity and survival: English longitudinal study of ageing. Am J Prev Med 2014:47:452-60.

28. Pukkala E, Verkasalo PK, Ojamo M, et al. Visual Impairment and cancer: a population based cohort study in Finland. Cancer Cause Control 1999;10:13-20.

29. Zhang X, Kahende J, Fan AZ, et al. Smoking and visual impairmentamong older adults with age related eye diseases. Prev Chronic Dis 2011;8:A84.

30. Anokye NK, Trueman P, Green C, et al. Physical activity and health related quality of life. BMC Public Health 2012;12:624.

31. Radak Z, Taylor AW, Ohno H, et al. Adaptation to exercise induced oxidative stress: from muscle to brain. Exerc Immunol Rev 2001:7:90-107.

32. Khandhadia S, Lotery A. Oxidation and age-related macular degeneration: insights from molecular biology. Expert Rev Mol Med 2010;12:28.

33. Mares JA, Voland RP, Sondel SA, et al. Healthy lifestyles related to subsequent prevalence of age-related macular degeneration. Arch Ophthalmo/ 2011;129:470-80.

34. Knudtson MD, Klein R, Klein BE. Physical activity and the 15-year cumulative incidence of age-related macular degeneration: the Beaver Dam Eye Study. Br J Ophthalmol 2006;90:1461-3.

35. Gopinath B, Liew G, Burlutsky G, et al. Physical activity and the 15year incidence of age-related macular degeneration. Invest Ophthalmol Vis Sci 2014;55:7799-803.

36. Lawson EC, Han MK, Sellers JT, et al. Aerobic exercise protects retinal function and structure from light-induced retinal degeneration. J Neurosci 2014;34:2406-12. 